\title{
APLICACIÓN DE LA REGLA FISCAL EN COSTA RICA
}

\section{APLICAÇÃO DA REGRA FISCAL NA COSTA RICA}

\section{Carlos Conejo Fernández ${ }^{1}$ David Cardoza Rodríguez ${ }^{2}$}

\begin{abstract}
Resumen
Costa Rica, ante el creciente deterioro de las finanzas públicas de los últimos diez años, aprobó una regla fiscal que liga el crecimiento del gasto público al crecimiento de la producción nacional y al nivel de la deuda pública. La regla se implementó a partir del año 2020. El objetivo de este artículo es resaltar el riesgo de llegar a resultados adversos si la regla fiscal se aplica de una forma que restringe de manera excesiva el gasto público. Respecto a la verificación del cumplimiento de la regla fiscal el Ministerio de Hacienda ha señalado que se debe utilizar como parámetro el presupuesto formulado para el año respectivo, mientras que la Contraloría General de la República sostiene que se debe utilizar el presupuesto efectivamente ejecutado. Se aplica un modelo de simulación lineal sencillo para calcular las posibles tendencias que seguirá el gasto público al utilizar cada uno de los parámetros indicados para su verificación. Se concluye que una interpretación muy restrictiva de la regla fiscal podría generar resultados contrarios a los buscados. Además, se señala que con la emergencia sanitaria causada por el Coronavirus la esta regla más bien constituye un instrumento que le resta grados de libertad al Gobierno, en momentos que requiere mayor flexibilidad en el manejo de la política fiscal.
\end{abstract}

Palabras clave: Coronavirus; déficit fiscal; deuda pública; finanzas públicas; política fiscal

Doi: https://doi.org/10.15359/eys.25-58.1

Recibido: 01-05-2020. Reenvíos: 04-05-2020, 15-06-2020, 22-06-2020, 29-06-2020. Aceptado: 29-06-2020. Publicado: 01-07-2020.

1 Doctor en Economía, docente e investigador del Proyecto de Estudios Fiscales de la Escuela de Economía de la Universidad Nacional, Costa Rica. Correo electrónico: carlos.conejo.fernandez@una.ac.cr ORCID https://orcid.org/0000-0001-9072-5328

2 Máster en Economía Internacional y Crecimiento Económico, docente e investigador del Proyecto de Estudios Fiscales de la Escuela de Economía de la Universidad Nacional, Costa Rica. Correo electrónico: david.cardoza.rodriguez@una.ac.cr ORCID https://orcid.org/0000-0002-2865-9697 


\begin{abstract}
As a consequence of the increasing deterioration of public finances in the last ten years, Costa Rica approved a fiscal rule that links growth of public spending with growth of national production and level of public debt. The rule was implemented in 2020 . The objective of this article is to highlight the risk of adverse results if the fiscal rule restricts public expenditure excessively. Regarding the verification of compliance with the fiscal rule, according to the Ministry of Treasury the budget formulated for the respective year should be used as a parameter, while according to the Comptroller General of the Republic what must be used is the budget effectively executed by each of the public entities. A simple linear simulation model is applied here to calculate the possible trends that public spending will follow when using each of the indicated parameters for verification. It is concluded that a very restrictive interpretation of the fiscal rule could generate unwanted results. In addition, it is pointed out that with the health emergency caused by the Coronavirus, the fiscal rule rather restricts the government's freedom when greater flexibility is needed to manage the fiscal policy.
\end{abstract}

Keywords: Coronavirus; fiscal deficit; public debt; public finances; fiscal policy

\title{
Resumo
}

Dada a crescente deterioração das finanças públicas nos últimos dez anos, a Costa Rica aprovou uma regra fiscal que vincula o crescimento dos gastos públicos ao crescimento da produção nacional e ao nível da dívida pública. A regra foi implementada a partir de 2020 . O objetivo deste artigo é destacar o risco de alcançar resultados adversos se a regra fiscal for aplicada de maneira a restringir excessivamente os gastos públicos. No que se refere à verificação do cumprimento da regra fiscal, o Ministério das Finanças indicou que o orçamento formulado para o respectivo ano deve ser usado como parâmetro, enquanto o Controlador Geral da República sustenta que o orçamento realmente executado deve ser utilizado. Este artigo aplica um modelo de simulação linear simples para calcular as possíveis tendências que os gastos públicos seguirão ao usar cada um dos parâmetros indicados para sua verificação. Conclui-se que uma interpretação muito restritiva da regra fiscal poderia gerar resultados contrários aos buscados. Além disso, ressalta-se que, com a emergência sanitária causada pelo coronavírus, a regra fiscal constitui um instrumento que reduz os graus de liberdade do governo, em momentos que exigem maior flexibilidade na gestão da política fiscal.

Palavras-chave: Coronavírus; déficit fiscal; dívida pública; Finanças públicas; política fiscal 


\section{Introducción}

La regla fiscal es un instrumento que busca ayudar a las economías a equilibrar las finanzas públicas y dar sostenibilidad a la deuda pública. Constituye una herramienta que favorece el logro de la consolidación fiscal, al limitar la posibilidad de que los gobiernos incurran en grandes desequilibrios fiscales, que se acumulan como una creciente deuda pública, la cual limita el desempeño económico del país.

Sin embargo, para lograr ese cometido la regla fiscal debe definirse con claridad y ser de fácil aplicación. Una regla confusa, con espacios para interpretación, o muy estricta, podría deteriorar, en vez de mejorar, la situación fiscal del país.

El objetivo de este artículo es resaltar el riesgo de que al ser aplicada la regla fiscal se genere un efecto adverso en la economía nacional, debido a la poca claridad respecto a la forma de evaluar su cumplimiento. Esta incertidumbre generó puntos de vista contrarios entre el Ministerio de Hacienda ( $\mathrm{MH})$, entidad encargada de formular y presentar el presupuesto nacional a la Asamblea Legislativa para ser aprobado, y la Contraloría General de la República (CGR), instancia encargada de velar por el adecuado uso de los recursos que administran las diferentes instancias del sector público costarricense. También se reflexiona sobre las implicaciones de la regla fiscal en el marco de la emergencia sanitaria causada por el Coronavirus ${ }^{3}$.

El trabajo consistió en identificar los aspectos principales de la regla fiscal respecto a su definición y cumplimiento, resaltar las posiciones del MH y de la CGR, la definición y aplicación de un modelo básico de simulación lineal para proyectar la posible tendencia que seguirá el gasto corriente del sector público, al aplicar ambos criterios para la evaluación del cumplimiento de la regla fiscal. Se concluye que una aplicación muy restrictiva de esta podría llevar a resultados opuestos a los buscados, que en vez de mejorar más bien tiende a empeorar la consolidación de las finanzas públicas del país en el mediano plazo.

El documento está estructurado de la siguiente manera: en el Apartado dos se hace una breve reseña histórica de la situación de las finanzas públicas en el país; en la sección tres se pone en contexto la regla fiscal que aprobó Costa Rica con la Ley n. ${ }^{\circ} 9635$; en el Apartado cuatro se esboza la posición del $\mathrm{MH}$ respecto a la verificación de la regla fiscal; en el Apartado cinco se plantea la posición de la CGR al respecto; en el seis se analiza la disyuntiva anterior; en la sección siete se cuantifica la posible tendencia que seguiría el gasto corriente del sector público no financiero, al utilizar ambas metodologías de verificación de cumplimiento de la regla fiscal; en el Apartado ocho se ubica la regla fiscal en el contexto de la emergencia causada por el Coronavirus; y en el último, se presentan las conclusiones.

3 Cabe señalar que el artículo fue escrito antes de iniciada la pandemia causada por el Coronavirus y su revisión se realizó ya iniciada la emergencia sanitaria; por eso se mantienen las proyecciones realizadas previo a la aparición de la pandemia y se realizan algunas reflexiones sobre los posibles efectos fiscales de la emergencia sanitaria. 


\section{Ubicación general de las finanzas públicas}

Las finanzas públicas de Costa Rica muestran un deterioro significativo desde la crisis del 2008. A partir de ese año la tasa de crecimiento de los ingresos fiscales se empezó a deteriorar y el gasto público empezó a crecer a un mayor ritmo, ya para el año 2016 el país tenía un déficit fiscal revelador, que siguió creciendo, como se muestra en la Tabla 1. El déficit financiero del Gobierno Central pasó de un 5,2 \% del PIB en el año 2016 a 7,0 $\%$ en el 2019. Este déficit se ha venido acumulando en forma de deuda pública la cual ha mostrado una tendencia creciente en los últimos años, como se muestra en la Tabla 1. La deuda del gobierno central como porcentaje del PIB pasó de 45,08\% en el 2016 a $58,48 \%$ en el 2019 y se proyecta que superará el $60 \%$ en el año 2020, como se muestra en los datos de la Tabla 1.

Tabla 1

Costa Rica: comportamiento de la producción y las finanzas públicas (2016-2019). Como porcentaje del PIB

\begin{tabular}{|c|c|c|c|c|c|}
\hline Indicador\año & 2016 & 2017 & 2018 & 2019 & $2020^{(1)}$ \\
\hline PIB real (tasa de crecimiento) & 4,2 & 3,4 & 2,6 & 2,1 & 2,5 \\
\hline Gasto del Gobierno Central & 19,9 & 20,5 & 20,2 & 20,9 & 20,7 \\
\hline Gasto corriente & 18,1 & 18,5 & 18,8 & 19,5 & 19,3 \\
\hline Déficit financiero & 5,2 & 6,1 & 5,9 & 7,0 & 6,9 \\
\hline Déficit primario & 2,4 & 3,0 & 2,3 & 2,2 & 1,3 \\
\hline Deuda del Gobierno Central & 45,08 & 48,41 & 53,17 & 58,48 & 61,0 \\
\hline
\end{tabular}

Proyecciones del BCCR realizadas antes de iniciar la pandemia causada por el Coronavirus. Fuente: Elaboración propia con datos del BCCR (2020a, págs. 7, 10 y 61); y MH. (2019, pág. 90).

El gasto corriente del Gobierno Central también mostró un crecimiento significativo en el periodo indicado, al pasar de un 18,1 \% del PIB en el año 2016 a un 19,5 \% en el año 2019, lo cual influye en el aumento del déficit fiscal y el endeudamiento público. Por otra parte, como se muestra en la Tabla 1, la producción nacional experimentó una desace-leración en los últimos años, ya que la tasa de crecimiento del PIB real pasó de $4,2 \%$ en el 2016 a 2,1 \% en el 2019, lo que afectó la recaudación y la consolidación fiscal del país.

Este deterioro en las finanzas públicas motivó a la Administración de Luis Guillermo Solís (2014-2018) a impulsar una reforma fiscal integral, la cual no fue aprobada en ese perio-do de gobierno, pero al final de su gestión quedó presentado en la Asamblea Legislativa un proyecto de reforma fiscal, el cual fue aprobado en diciembre de 2018, cuando ya había asumido la presidencia el señor Carlos Alvarado, quien se había comprometido en la campaña electoral a trabajar por la mejora de las finanzas públicas. 


\section{Contexto de la regla fiscal en Costa Rica}

El 3 de diciembre de 2018 la Asamblea Legislativa de Costa Rica aprobó la Ley de Fortalecimiento de las Finanzas Públicas (Ley . $^{\circ} 9635$ ), la cual incluye, en el título IV, una regla fiscal de gasto, que cubre al sector público no financiero. La aplicación de la regla fiscal rige a partir del año 2020. Ante el creciente deterioro que han venido experimentado las finanzas públicas del país desde el año 2008, la nueva ley, y en particular la regla fiscal, pretende apoyar la consolidación fiscal y lograr la sostenibilidad de la deuda pública en el mediano plazo.

El artículo 10 del Título IV de la Ley $n .^{\circ} 9635$, respecto a la regla fiscal, establece que el crecimiento del gasto corriente de las entidades del sector público no financiero estará determinado por dos variables: a) el nivel de deuda del Gobierno Central como porcentaje del Producto Interno Bruto (PIB) y; b) el crecimiento promedio del PIB nominal de los cuatro años anteriores al año de formulación del presupuesto nacional. El artículo 11 del Título IV de la Ley $n .^{\circ} 9635$ determina los siguientes rangos de deuda que deben considerarse para calcular el crecimiento del gasto corriente (ver Tabla 2).

Tabla 2

Costa Rica: Rangos de deuda para calcular el crecimiento del gasto corriente

\begin{tabular}{lll}
\hline Escenario & Deuda/PIB del año anterior $\left(\mathbf{d}_{\mathrm{t}-1}\right)$ & Crecimiento del gasto* \\
\hline A & $<30 \%$ & $100 \%$ \\
$B$ & $30 \% \leq \mathrm{d}_{\mathrm{t}-1}<45 \%$ & $85 \%$ \\
$\mathrm{C}$ & $45 \% \leq \mathrm{d}_{\mathrm{t}-1}<60 \%$ & $75 \%$ \\
$D$ & $\mathrm{~d}_{\mathrm{t}-1} \geq 60 \%$ & $65 \%$ \\
\hline
\end{tabular}

\footnotetext{
*Se refiere al límite máximo permitido de crecimiento del gasto corriente de las instancias del sector público no financiero, respecto al crecimiento promedio del PIB nominal de los últimos cuatro años anteriores al año de formulación del presupuesto.

Fuente: Elaboración propia con base en el artículo 11 del título IV de la Ley n9635.
}

La normativa también señala que, si la deuda pública supera el $60 \%$ de la producción nacional, la regla fiscal se aplica al incremento del gasto total, no solo al gasto corriente. Incluye también los gastos de capital, limitando las posibilidades de promover la inversión pública, justo en momentos en que el país cuenta con un rezago significativo en esta área.

Respecto a la verificación del cumplimiento de la regla fiscal, ha surgido una diferencia de criterio entre el MH y la CGR. El Ministerio considera que implementar y verificar la regla fiscal debe realizarse con base en el presupuesto formulado, mientras que la Contraloría sostiene que debe utilizarse como base de comparación el presupuesto ejecutado o liquidado de cada una de las entidades. Veamos el planteamiento de cada una de las instancias presentadas. 


\section{Posición del Ministerio de Hacienda}

El MH sostiene que la verificación del cumplimiento de la regla fiscal en cada una de las entidades del sector público no financiero debe realizarse con base en el presupuesto formulado. Considera que la regla fiscal establecida en la Ley $n .^{\circ} 9635$ es una regla de gasto corriente de los presupuestos, así redactado en el artículo 11 de la norma; de manera que su cálculo y verificación deben realizarse con base en el presupuesto formulado, y no sobre la base del presupuesto ejecutado. Además, resalta que si se utiliza el presupuesto ejecutado podría llevar a un estancamiento en el gasto corriente, y que ese no es el espíritu de la ley.

También señala dicho Ministerio que la Dirección General de Presupuesto Nacional (DGPN) utilizará como variables para verificar el cumplimiento de la regla fiscal en el 2020, según el artículo 21 del Título IV de la referida Ley, el gasto corriente presupuestario inicial del periodo previo, el gasto corriente presupuestario inicial y definitivo del periodo en que se aplica la regla, así como la tasa de crecimiento máxima autorizada para dicho gasto.

Por otro lado, cabe resaltar que el Reglamento al Título IV de la Ley N. ${ }^{\circ} 9635$, en su artículo 3 indica lo siguiente: "... para la aplicación de la regla fiscal, las entidades deberán tomar como referencia el gasto corriente incorporado en el presupuesto ordinario aprobado del año en que se realiza la formulación del presupuesto del siguiente periodo..." (Asamblea Legislativa, pág. 5).

Es decir, el reglamento señala que para aplicarse la regla fiscal se debe tomar como referencia el presupuesto formulado, aspecto que no es compartido por la CGR.

\section{Posición de la Contraloría General de la República}

La CGR (2020) señala que la verificación del cumplimiento de la regla fiscal debe realizarse con base en el presupuesto ejecutado. La CGR resalta que existen tres momentos diferentes para la verificación del cumplimiento: la formulación presupuestaria (art. 19), el monitoreo trimestral de la ejecución presupuestaria (art. 20) y la comprobación final, el cual se realizará con base en los datos de la liquidación presupuestaria, (art. 21). La CGR sostiene que el proceso presupuestario pleno y efectivo se da con la liquidación presupuestaria, y que por tanto la variable que se debe utilizar para la verificación del cumplimiento de la regla fiscal es el presupuesto ejecutado y no el presupuesto formulado. Además, resalta que, si se utiliza el presupuesto formulado, se extendería de manera significativa el periodo de tiempo para poder estabilizar la deuda pública, que es uno de los principales objetivos buscados con la aplicación de la regla fiscal.

Como ejemplo para aclarar la situación, la CGR compara el nivel de gasto corriente permitido por la regla fiscal para los años 2019 y 2020, que utiliza, por un lado, el presupuesto formulado (posición de $\mathrm{MH}$ ) y por otro lado, el presupuesto ejecutado (posición de la CGR), como se resume en la Tabla 3. 
Tabla 3

Costa Rica. Verificación de la regla fiscal sobre el gasto corriente. (En millones de colones)

\begin{tabular}{llrrr}
\hline Criterio & Variable a utilizar & Regla fiscal & \multicolumn{1}{c}{ Año 2019 } & \multicolumn{1}{c}{ Año 2020 } \\
\hline MH & Presupuesto formulado & $4,67 \%$ & $\$ 7539952$ & $\$ 7892068$ \\
CGR & Presupuesto ejecutado & $4,67 \%$ & $\$ 7126144$ & $\$ 7458935$ \\
& Diferencia para el 2020 & & & $\$ 433133$ \\
\hline
\end{tabular}

Fuente: Elaboración propia con datos de CGR.

El ejercicio anterior aplica un 4,67\% de incremento al presupuesto formulado y ejecutado en el 2019 para calcular el límite máximo de gasto permitido por la regla fiscal para el año 2020. La última columna de la Tabla 2 muestra el tope máximo de gasto corriente permitido según el criterio del MH y según el criterio de la CGR, donde se evidencia que el tope del MH es superior en $\$ 433133$ millones, lo cual representa alrededor de 1,13\% del PIB.

\section{Análisis de la disyuntiva presentada ente el Ministerio de Hacienda y la Contraloría}

La regla fiscal tiene como objetivo consolidar las finanzas públicas, asegurando la sostenibilidad de la deuda pública, manteniendo como prioridad el fomento de la inversión y el crecimiento de la producción nacional. Para ello se utilizan como variables el gasto corriente, la tasa de crecimiento del PIB y la deuda pública. El método consiste en limitar el aumento del gasto corriente de las instancias del sector público no financiero a un porcentaje del crecimiento promedio del PIB de los cuatro años anteriores, el cual tiene como referencia el nivel de la deuda del Gobierno Central.

Lo que se busca al implementar la regla fiscal es que el gasto corriente (o el gasto total si la relación deuda/PIB supera el 60 \%) crezca apenas en una proporción del crecimiento promedio del PIB nominal de los últimos cuatro años. El objetivo de la aplicación de la regla no es que el gasto se estabilice y menos que se reduzca. De hecho, la misma ley señala en el artículo 16.b, que, si el PIB crece menos del $1 \%$, se suspende la aplicación de la regla fiscal, ya que implicaría un crecimiento muy bajo del gasto corriente. Aplicar la regla de una manera que detenga el crecimiento del gasto corriente no es el espíritu de la ley, y peor aún, podría ir en contra del objetivo buscado que es la consolidación fiscal y el crecimiento económico del país. Sin embargo, se podría decir que sí hay una intencionalidad de recortar el gasto corriente como porcentaje del PIB, en el mediano y largo plazo.

Si se utiliza el presupuesto ejecutado como variable para la verificación del cumplimiento de la regla fiscal se podría estar poniendo una restricción excesiva al crecimiento del gasto corriente, con los efectos adversos que esto genera en la producción, el empleo, el déficit fiscal y el mismo endeudamiento público. De hecho, el ejercicio realizado por la CGR y mostrado en la Tabla 3, señala que el límite máximo de gasto corriente permitido para el año 2020 (\$7 458935 millones) sería inferior al del año 2019 (\$7 539952 millones). Es decir, se estaría dando un decrecimiento en el límite máximo permitido de gasto

Carlos Conejo Fernández • David Cardoza Rodríguez 
corriente nominal para el sector público no financiero. Esto podría deprimir aún más la actividad económica, con los efectos adversos que ello genera en las finanzas públicas.

Para profundizar en lo señalado anteriormente, se emplea un modelo de simulación lineal sencillo para visualizar la posible tendencia que seguiría el límite máximo de gasto permitido, al utilizar para la verificación de la regla fiscal, por un lado, el presupuesto formulado y por otro lado el presupuesto ejecutado. Los resultados se muestran en la Tabla 4. El ejercicio se construye con base en los siguientes supuestos:

- Ejecución presupuestaria del gasto corriente: $94 \%$, que se mantiene constante 4

- Porcentaje máximo de crecimiento del gasto corriente permitido por la regla fiscal: 4,67 \%, la que rige para el periodo 2019-2020, que se mantiene constante a lo largo del periodo bajo análisis

- Presupuesto inicial: año 2019=100, para facilitar la interpretación de los datos

Tabla 4

Costa Rica: Ejercicio de simulación de la aplicación de la regla fiscal

\begin{tabular}{|c|c|c|c|c|c|c|}
\hline & 2019 & 2020 & 2021 & 2022 & 2023 & 2024 \\
\hline \multicolumn{7}{|l|}{$\begin{array}{l}\text { Aplicación sobre presupuesto } \\
\text { formulado }\end{array}$} \\
\hline Gasto total presupuestado & 100 & 104,67 & 109,56 & 114,67 & 120,03 & 125,64 \\
\hline Regla fiscal aplicada & & $4,67 \%$ & $4,67 \%$ & $4,67 \%$ & $4,67 \%$ & $4,67 \%$ \\
\hline Crecimiento del gasto (\%) & & $4,67 \%$ & $4,67 \%$ & $4,67 \%$ & $4,67 \%$ & $4,67 \%$ \\
\hline \multicolumn{7}{|l|}{$\begin{array}{l}\text { Aplicación sobre presupuesto } \\
\text { ejecutado }\end{array}$} \\
\hline Gasto total presupuestado & 100 & 98,39 & 96,81 & 95,25 & 93,71 & 92,20 \\
\hline Porcentaje de ejecución & $94 \%$ & $94 \%$ & $94 \%$ & $94 \%$ & $94 \%$ & $94 \%$ \\
\hline Regla fiscal aplicada & $4,67 \%$ & $4,67 \%$ & $4,67 \%$ & $4,67 \%$ & $4,67 \%$ & $4,67 \%$ \\
\hline Crecimiento del gasto (\%) & & $-1,61 \%$ & $-1,61 \%$ & $-1,61 \%$ & $-1,61 \%$ & $-1,61 \%$ \\
\hline
\end{tabular}

Fuente: Elaboración propia con datos del Banco Central, 2020.

Los datos de la Tabla 4 muestran que si el límite de crecimiento al gasto corriente permitido por la regla fiscal $(4,67 \%)$ se aplica al presupuesto formulado en el año anterior, el monto presupuestado crecerá, con base en el crecimiento del PIB nominal y la restricción del nivel de deuda establecido en el artículo 11 de la Ley, pasando de 100 en el año 2019 a 125,64 en el año 2024. Siempre habrá un aumento del gasto corriente presupuestado, salvo que el PIB decrezca. Pero en ese último caso, la misma ley señala que cuando la proyección de crecimiento del PIB sea inferior al $1 \%$ se puede suspender la aplicación de la regla fiscal.

4 Según la CGR, el promedio de la ejecución del gasto corriente del periodo 2007-2019 fue del 94 \% (CGR, 2020 pág. 4). 
Por otro lado, si se aplica la regla fiscal con base en el presupuesto ejecutado, se observa que el nivel de gasto permitido más bien tiende a la baja, en este caso decrece en un 1,61 \% de un año a otro, pasando de 100 en el año 2019 a 92,20 en el 2024; una reducción del 7,8 \% en un periodo de cinco años.

Cabe señalar que, en general, si el porcentaje de subejecución del presupuesto del año base es superior al porcentaje de crecimiento que establece la regla fiscal, el límite máximo de gasto permitido tenderá a la baja. Solo si la regla fiscal es superior al nivel de subejecución presupuestaria el límite máximo de gasto permitido crecerá; pero lo haría a un nivel muy reducido, siendo más pequeño entre más cercanas están esas variables. ${ }^{5}$ Nótese que, con base en los datos históricos de ejecución presupuestaria del país, lo esperable es que el monto nominal de gasto corriente permitido tienda a bajar en el tiempo, si se aplica la regla fiscal con base en el presupuesto ejecutado.

La restricción del gasto se agudizaría si la relación deuda/PIB supera el $60 \%$, ya que en ese caso la regla fiscal se aplica al gasto total del sector público no financiero, que incluye también los gastos de capital. Esto limitaría el accionar del gobierno, justo en momentos en que más se requiere el impuso a la inversión pública, por los siguientes aspectos: a) el país arrastra un rezago muy significativo en inversión pública, en particular en infraestructura vial y educativa; b) la producción nacional ha venido creciendo a tasas cada vez menores, como se indicó en el Apartado 1, de ahí la necesidad de impulsar la inversión pública como un motor de crecimiento económico, y c) la emergencia sanitaria causada por el Coronavirus ha puesto en evidencia la necesidad de realizar fuertes inversiones públicas en el sector salud.

Ahora bien, de aquí podría inferirse que ese resultado es el idóneo, pues lo que se busca es reducir el gasto público para así reducir la deuda; pero esa interpretación podría ser engañosa, ya que la reducción del gasto corriente del sector público tiene efectos adversos en la producción nacional y la recaudación de impuestos, lo que puede empeorar en vez de mejorar la situación fiscal del país.

\section{Posibles efectos del Decreto Ejecutivo 42218-H}

Ante la disyuntiva entre el MH y la CGR, el Banco Central de Costa Rica (BCCR) presentó una interpretación ${ }^{6}$ "intermedia” que ha sido retomada por Casa Presidencial de Costa

5 Por otro lado, si para la elaboración del presupuesto se utiliza como base el presupuesto formulado en el año anterior, más lo que establece la regla fiscal, y así se aprueba por las instancias competentes (Asamblea Legislativa o CGR), pero la verificación de cumplimiento a posteriori se realiza con base en el presupuesto ejecutado, implicaría que la instancia cuenta con un presupuesto formalmente aprobado, pero que no puede gastar en su totalidad, ya que incumpliría la regla fiscal.

Otra dificultad de utilizar el presupuesto liquidado es que el presupuesto se aprueba en setiembre de cada año, mes en el cual aún no se conoce cuál va a ser el presupuesto ejecutado en ese año. De manera que habría que aprobar un presupuesto con una proyección de gasto, el cual deberá ser ajustado a inicios del año siguiente, una vez que se tenga certeza del presupuesto efectivamente gastado el año precedente. Lo anterior requerirá una modificación presupuestaria de cada una de las instancias públicas, con todos los trámites que ello implica, haciendo más complejo y menos confiable la aplicación de la regla fiscal.

6 BCCR. 19 de febrero de 2020a. Aplicación de la Regla Fiscal, comunicado de prensa, oficio CPBCCR-004-2020, San José, Costa Rica.

Carlos Conejo Fernández • David Cardoza Rodríguez 
Rica (2020), quien emitió un decreto ${ }^{7}$ sobre la implementación de la regla fiscal. El decreto señala que para la presupuestación se debe utilizar como referencia el presupuesto formulado en el año anterior, mientras que para la verificación de cumplimiento de la regla fiscal se debe utilizar como referencia el presupuesto ejecutado en el año anterior. Es decir, el decreto señala que se deben utilizar las dos variables, pero en momentos y para fines distintos.

Si bien el Decreto Ejecutivo 42218-H señala la forma de aplicar la regla fiscal, que sería en dos niveles, aún queda gran incertidumbre sobre su implementación efectiva. Lo anterior debido a que el presupuesto formulado podría seguir creciendo sin que las entidades del sector público tengan una posibilidad real de ejecutarlo en su totalidad, ya que incumplirían la regla fiscal, situación que se verificaría a posteriori al comparar los presupuestos efectivamente gastados. También es importante resaltar que en la práctica es difícil para las entidades del sector público ejecutar el cien por ciento de sus presupuestos, por el riesgo de sobrepasar el límite de gasto permitido por la regla fiscal, aunque sí podría lograrse una mejora en la ejecución presupuestaria de las diferentes instancias del sector público no financiero, con la implementación de la regla fiscal.

Para profundizar en este análisis se realiza el siguiente ejercicio de simulación, con el objetivo de visualizar la posible tendencia del gasto efectivo, al aplicar lo que establece el Decreto Ejecutivo 42218-H; es decir, al utilizar para el proceso de presupuestación el monto del presupuesto formulado en el año anterior y para la verificación de cumplimiento se utiliza el presupuesto ejecutado. Los datos se muestran en la Tabla 5.

La Tabla 5 se construye a partir de un presupuesto de 100 en el año 2019 y con un crecimiento del gasto público definido por la regla fiscal del 4,67 \% para el 2020 (porcentaje aplicado ese año), y para los años siguientes se define con base en las proyecciones de crecimiento del PIB nominal elaboradas por el MH (2019, pág. 13). Además, para los años 2022-2024 se supone una relación deuda/PIB superior al $60 \%$, de manera que el valor de la regla fiscal será del $65 \%$ del crecimiento promedio del PIB nominal de los cuatro años anteriores.

En este ejercicio, para la verificación del cumplimiento de la regla fiscal, se plantean cuatro escenarios con diferentes niveles de ejecución presupuestaria, para el periodo 20192024. Los niveles de esta ejecución, utilizados para el cálculo del crecimiento del gasto corriente (junto con el crecimiento del PIB y la relación deuda/PIB), son los siguientes: $94 \%^{8}, 95 \%, 96 \%$ y $97 \%^{9}$.

A partir de los supuestos anteriores, la formulación presupuestaria crecería de forma sostenida en el periodo, pasando de 100 en el 2019 a 121,25 en el 2024, es decir, un crecimiento del $21,25 \%$ en el periodo (primera fila de la Tabla 5).

7 Decreto Ejecutivo 42218-H, del 26-2-2020.

8 La ejecución promedio del gasto corriente del Gobierno Central en el periodo 2007-2019 fue del $94 \%$, según lo señalado en el oficio de la CGR, DFOE-SAF-0073 (2020, pág. 4).

9 Es de esperar que con la implementación de la regla fiscal las instancias mejoren la ejecución presupuestaria. 
Por su parte el límite máximo de gasto permitido en la verificación ex post de la regla fiscal se determina al comparar el nivel de gasto ejecutado por las instancias en el año respectivo con el del año anterior. Dado el valor de la esta regla, entre menor sea la ejecución presupuestaria menor será el nivel de gasto permitido.

Como se muestra en la Tabla 5, si la ejecución presupuestaria es de un $94 \%$, el nivel de gasto permitido más bien tiende a la baja, pasando de 100 en el año 2019 a 88,99 en el 2024, es decir una reducción del $11 \%$ en el periodo indicado. De igual forma, si el porcentaje de ejecución presupuestaria es de un $95 \%$ o $96 \%$ el nivel de gasto máximo permitido no crece y más bien se reduce ligeramente. Solo si el porcentaje de ejecución presupuestaria es de $97 \%$, el nivel de gasto máximo permitido tiende a crecer de forma leve, al pasar de 100 en el año 2019 a 104,12 en el año 2024, como se muestra en la Tabla 5. Este nivel de crecimiento es muy bajo si se compara con el crecimiento anual promedio del gasto corriente del Gobierno Central de Costa Rica en los últimos años.

Tabla 5

Costa Rica: Ejercicio de simulación de la aplicación de la regla fiscal (con distintos porcentajes de ejecución presupuestaria)

\begin{tabular}{lcccccc}
\hline & $\mathbf{2 0 1 9}$ & $\mathbf{2 0 2 0}$ & $\mathbf{2 0 2 1}$ & $\mathbf{2 0 2 2}$ & $\mathbf{2 0 2 3}$ & $\mathbf{2 0 2 4}$ \\
\hline Formulación & & & & & & \\
Gasto total a presupuestar & 100 & 104,67 & 108,99 & 112,91 & 116,86 & 121,25 \\
Regla fiscal aplicada & & $4,67 \%$ & $4,13 \%$ & $3,59 \%$ & $3,50 \%$ & $3,76 \%$
\end{tabular}

\section{Verificación (con 94 \% de ejecución presupuestaria)}

\begin{tabular}{|c|c|c|c|c|c|c|}
\hline Ejecución /evaluación & 100 & 98,39 & 96,31 & 93,78 & 91,24 & 88,99 \\
\hline Regla fiscal aplicada & & $4,67 \%$ & $4,13 \%$ & $3,59 \%$ & $3,50 \%$ & $3,76 \%$ \\
\hline Crecimiento del gasto (\%) & & $-1,61$ & $-2,12$ & $-2,63$ & $-2,71$ & $-2,47$ \\
\hline \multicolumn{7}{|c|}{ Verificación (con 95 \% de ejecución presupuestaria) } \\
\hline Ejecución /evaluación & 100 & 99,44 & 98,37 & 96,80 & 95,18 & 93,82 \\
\hline Regla fiscal aplicada & & $4,67 \%$ & $4,13 \%$ & $3,59 \%$ & $3,50 \%$ & $3,76 \%$ \\
\hline Crecimiento del gasto (\%) & & $-0,56$ & $-1,08$ & $-1,59$ & $-1,68$ & $-1,43$ \\
\hline \multicolumn{7}{|c|}{ Verificación (con 96 \% de ejecución presupuestaria) } \\
\hline Ejecución /evaluación & 100 & 100,48 & 100,45 & 99,89 & 99,25 & 98,86 \\
\hline Regla fiscal aplicada & & $4,67 \%$ & $4,13 \%$ & $3,59 \%$ & $3,50 \%$ & $3,76 \%$ \\
\hline Crecimiento del gasto (\%) & & 0,48 & $-0,04$ & $-0,55$ & $-0,64$ & $-0,39$ \\
\hline \multicolumn{7}{|c|}{ Verificación (con 97\% de ejecución presupuestaria) } \\
\hline Ejecución /evaluación & 100 & 101,53 & 102,55 & 103,05 & 103,45 & 104,12 \\
\hline Regla fiscal aplicada & & $4,67 \%$ & $4,13 \%$ & $3,59 \%$ & $3,50 \%$ & $3,76 \%$ \\
\hline Crecimiento del gasto (\%) & & 1,53 & 1,01 & 0,48 & 0,39 & 0,65 \\
\hline
\end{tabular}

Fuente: Elaboración propia con datos del BCCR y del MH. 
La situación se vuelve más compleja al superar la deuda del Gobierno Central el 60 $\%$ del PIB, lo cual limita el crecimiento de la inversión pública, lo que incide en el crecimiento del PIB y la recaudación fiscal ${ }^{10}$, incrementa el déficit fiscal ${ }^{11}$ y el endeudamiento público. El efecto recesivo será mayor entre más grande sea la subejecución presupuestaria y entre menor sea la regla fiscal ${ }^{12}$. De manera que el resultado final podría ser contrario al esperado y llevar a un incremento de la deuda pública como porcentaje de la producción nacional.

Cabe señalar que el BCCR, al considerar los efectos económicos de la pandemia sanitaria causada por el Coronavirus, proyecta una reducción de la producción nacional de un 3,6 \% para el año 2020, (Estrada, 2020), lo cual agravaría aún más la situación de las finanzas públicas. Lo anterior implica que los resultados del modelo anterior son conservadores ya que, si la producción se reduce, el límite de gasto permitido por la regla fiscal en los años siguientes también será menor, lo que restringiría, por esa vía, el gasto público y la actividad económica.

\section{La regla fiscal y el Coronavirus}

La Organización Mundial de la Salud (OMS), ha declarado al Coronavirus como una pandemia mundial. Los efectos de este flagelo sobre la salud humana y las economías de los diferentes países son de dimensiones gigantescas, impensables antes de iniciada la propagación del virus.

La emergencia ha cambiado las prioridades de las políticas públicas. Al respecto, Thomsen (2020) señala que los países europeos más desarrollados han implementado políticas monetarias y fiscales expansivas para contener el impacto de la crisis. Los límites de las reglas fiscales han sido suspendidos para facilitar el apoyo a la emergencia, y se ha vuelto a permitir el surgimiento del déficit fiscal. Asimismo, los bancos centrales han lanzado amplios programas de compra de títulos... (p. 1)

Para hacer frente a la pandemia, los países se han visto en la necesidad de implementar medidas fiscales que permitan "reorganizar presupuestos para implementar paquetes de estímulo fiscal a fin de fortalecer los sistemas de salud, proteger los ingresos y minimizar la contracción de la economía". (Comisión Económica para América Latina y el Caribe, 2020, pág. 46)

El Coronavirus ha hecho necesario otorgar mayor flexibilidad a los Gobiernos para reorganizar y ampliar los presupuestos, permitir de forma temporal la desgravación fiscal, subsidiar a la población que enfrenta dificultades laborales, y evitar el deterioro del tejido productivo. Aquí se requiere contar con financiamiento externo y la implementación

10 Según Barreix \& Corrales (2019, pág. 8), la evidencia para América Latina señala que cada vez que el PIB decrece, la recaudación fiscal decrece en una mayor proporción. Es decir, que la elasticidad de esta respecto al PIB es mayor que uno.

11 El BCCR, (2020a, pág. 91), proyectó en enero de 2020 un déficit fiscal del 5,9 \% y 5,7 \% para los años 2020 y 2021 , respectivamente. Sin embargo, a consecuencia de la pandemia causada por el Coronavirus, el $\mathrm{MH}$ lo proyecta en $8,6 \%$ para el 2020.

12 Aquí no se están cuantificando los efectos de la emergencia sanitaria sobre la actividad económica.

Carlos Conejo Fernández • David Cardoza Rodríguez 
de políticas fiscales y monetarias expansivas. El BCCR tiene un margen de acción importante y está llamado a apoyar al Gobierno y al Sistema Financiero Nacional en momentos que podría experimentar un crecimiento abrupto en los niveles de morosidad. Según Corrales (2020, pág. 1), las políticas del BCCR deberán siempre estar apegadas a criterios técnicos y a la legislación nacional pertinente.

Ante la emergencia causada por el Coronavirus, el Gobierno de la República creó un fondo especial de un billón de colones para contar con liquidez y así poder hacer frente a la emergencia de manera oportuna. Estos recursos representan alrededor de un $3 \%$ del PIB y provienen del proyecto "Ley Pagar" que es una contribución de distintas instancias públicas (\$226 mil millones), Banco de Desarrollo de América Latina (CAF) (\$287 mil millones), un empréstito del Banco Interamericano de Desarrollo (BID)-Agencia Francesa de Desarrollo (AFD) (\$216 mil millones) y del proyecto de titularización de utilidades del Instituto Nacional de Seguros (INS) (\$288 mil millones) (Oviedo, E., 2020). Si bien el monto de este fondo es significativo, resulta a todas luces insuficiente, dada la gravedad de los efectos económicos y sociales de la pandemia.

La emergencia sanitaria implicará un amplio deterioro de las finanzas públicas. El Gobierno recaudará menos y gastará más. El déficit fiscal se ensanchará y la deuda pública seguirá creciendo. El ministro de Hacienda estima para el año 2020 el déficit fiscal será de un $8,6 \%$ del PIB y que la deuda del Gobierno Central podría alcanzar el $67 \%$ de la producción nacional (Leitón, P., 2020). Sin embargo, no hay mayores alternativas, ya que los objetivos de atención a la emergencia sanitaria se anteponen a los objetivos de equilibrio fiscal y sostenibilidad de la deuda pública.

Cabe señalar que el artículo 16 del Título IV de la ley n. ${ }^{\circ} 9635$ establece la posibilidad de dejar sin efecto la regla fiscal, hasta por un periodo de tres años, cuando el país es declarado en emergencia nacional y que la atención de esa emergencia requiera de una erogación de gasto corriente superior al 0,3\% del PIB; o cuando se tengan proyecciones de crecimiento económico inferiores al $1 \%$. Basado en esta cláusula el Gobierno, a finales de abril de 2020, notificó a la Asamblea Legislativa, que la regla fiscal contenida en la Ley de Fortalecimiento de las Finanzas Públicas (Ley N. ${ }^{\circ}$ 9635) fue levantada para cuatro instituciones que están en la atención de la emergencia por el Coronavirus. Se trata del Instituto Mixto de Ayuda Social (IMAS), el Ministerio de Trabajo y Seguridad Social (MTSS), el Cuerpo de Bomberos de Costa Rica y el Instituto Costarricense de Investigación y Enseñanza en Nutrición y Salud (Inciensa).

En este contexto, las municipalidades del país impulsaron una iniciativa de ley que fue aprobada por la Asamblea Legislativa en mayo del 2020, con la cual fueron excluidas de la aplicación de la regla fiscal. Las municipalidades justificaron, entre otros aspectos, que esta regla les resta grados de libertad en la atención de la emergencia sanitaria. Este escenario constituye un antecedente para que otras instancias del sector público soliciten el mismo beneficio.

Se evidencia que, en circunstancias como estas, la regla fiscal constituye una restricción, una camisa de fuerza, en momentos en que el Gobierno requiere de más flexibilidad

Carlos Conejo Fernández • David Cardoza Rodríguez 
para atender la situación precaria por la que atraviesa el país. Pero a la vez la regla fiscal constituye un norte para asegurar la sostenibilidad de las finanzas públicas en el largo plazo. De ahí que el país enfrenta una disyuntiva compleja, por un lado, tomar las medidas pertinentes para asegurar el menor impacto posible del Coronavirus, y por otro lado, evitar que las finanzas públicas se salgan de control.

\section{Conclusiones}

La regla fiscal aprobada en diciembre de 2018 y que entró a regir a partir del 2020 tiene como objetivo favorecer la consolidación fiscal y la sostenibilidad de la deuda pública costarricense en el mediano y largo plazo. La efectividad de este instrumento de política fiscal dependerá, en gran parte, de la forma en que se implemente, así como de los criterios y procedimientos utilizados para su verificación.

Ha existido una diferencia de criterio entre el $\mathrm{MH}$, quien sostiene que para la verificación de la regla fiscal debe utilizarse el presupuesto formulado en el año anterior, y la CGR, quien señala que debe utilizarse el presupuesto ejecutado. Ante esta disyuntiva el Casa Presidencial de Costa Rica (2020) emitió el decreto № 42218-H, el cual indica que en el proceso de presupuestación se debe utilizar el presupuesto formulado en el año anterior, mientras que en la verificación se debe utilizar el presupuesto ejecutado. Es decir, se deben utilizar ambas variables una, ex ante en el proceso de formulación presupuestaria y la otra, ex post en la evaluación final del cumplimiento de la regla fiscal. Sin embargo, ambos sistemas llevan a resultados distintos, ya que el criterio de la Contraloría es más restrictivo que el que aplicará el $\mathrm{MH}$, debido a que las instancias del sector público no logran ejecutar el $100 \%$ del presupuesto formulado. De manera que persiste cierta incertidumbre respecto a la forma efectiva en que se aplicará la regla fiscal en el país, lo cual vulnera la confianza de las personas respecto a la efectividad de la aplicación de la regla fiscal.

A partir del ejercicio de simulación desarrollado en este artículo se desprende que, si la verificación de la regla fiscal se realiza con base en el presupuesto formulado, como sugiere el $\mathrm{MH}$, el crecimiento del gasto corriente seguirá una tendencia moderada, acorde con los objetivos plateados en esta regla, de consolidación fiscal en el mediano plazo.

De las proyecciones desarrolladas en este artículo se desprende que, si la verificación de la regla fiscal se realiza con base en el presupuesto ejecutado, se limitaría de manera significativa el crecimiento del gasto del sector público costarricense, el cual podría tender a un crecimiento nulo o incluso negativo. El menor gasto corriente restringe la tasa de crecimiento del PIB y la recaudación fiscal. Lo anterior podría incrementar el déficit fiscal y, por tanto, la necesidad de recurrir a más (no menos) endeudamiento público, lo que dificultaría el logro de los objetivos buscados al aplicar la regla fiscal.

La verificación de la regla fiscal, con base en el presupuesto ejecutado, podría implicar una aplicación muy restrictiva de la normativa, lo que puede afectar el adecuado funcionamiento de la institucionalidad pública, al limitar su capacidad para atender de forma adecuada los diferentes servicios que presta el Gobierno a la ciudadanía, particularmente 
los programas sociales. Lo anterior podría vulnerar la credibilidad de la ciudadanía en las bondades de dicha regla y buscar su no aplicación. Las municipalidades ya lograron la aprobación de una ley que las exime en este sentido, y otras instancias podrían perseguir el mismo beneficio.

La emergencia por el Coronavirus ha implicado un cambio en los objetivos de la política fiscal en el país y el mundo. La prioridad se ha trasladado a la atención de la emergencia sanitaria y a dar apoyo a la población que ha visto disminuidos o esfumados sus ingresos, y en ese sentido, los objetivos de equilibrio fiscal han pasado a segundo plano. La atención de la pandemia ha llevado al Gobierno a levantar la aplicación de la regla fiscal en las instancias encargadas de atender la emergencia causada por el Coronavirus.

El actual deterioro de las finanzas públicas permite inferir que para el 2020 la deuda del Gobierno Central superará el 60 \% del PIB, nivel a partir del cual la regla fiscal se aplica al gasto total del sector público no financiero, no solo al gasto corriente, situación que implica una mayor rigidez a la administración pública, en momentos que se requiere de mayor flexibilidad del gasto para atender la crisis sanitaria y económica por la que atraviesa el país.

Es un momento propicio para abrir un espacio de diálogo nacional con visión prospectiva para analizar los retos y bondades que implica la implementación de la regla fiscal, en el marco de la sostenibilidad de las finanzas públicas del país.

\section{Referencias}

Asamblea Legislativa. (2018). Ley 9635, de Fortalecimiento de las Finanzas Públicas. Recuperado de http://www.pgrweb.go.cr/scij/Busqueda/Normativa/Normas/nrm_norma. aspx?param1=NRM\&nValor1=1\&nValor2=87720\&nValor3=118801\&strTipM=FN

Barreix, A., \& Corrales, L. (2019). Reglas Fiscales Resilientes en América Latina. Doi: http://dx.doi. org/10.18235/0002003

Banco Central de Costa Rica. (2020a). Programa Macroeconómico 2020-2021. Recuperado de https://activos.bccr.fi.cr/sitios/bccr/publicaciones/DocPolticaMonetarialnflacin/Programa_Macroeconomico_2020-2021.pdf

Banco Central de Costa Rica. (2020b). Aplicación de la Regla Fiscal. En Comunicados de Prensa. Recuperado de https://www.bccr.fi.cr/seccion-comunicados-de-prensa/ comunicados-de-prensa

Comisión Económica para América Latina y el Caribe. (2020). Coyuntura, escenarios y proyecciones hacia 2030 ante la presente crisis de Covid-19. En Presentaciones. Recuperado de https://www.cepal.org/es/presentaciones/ coyuntura-escenarios-proyecciones-2030-la-presente-crisis-covid-19

Contraloría General de la República. (2020). Ampliación de criterio sobre expediente legislativo N. ${ }^{\circ}$ 21.573. En Oficio DFOE-SAF-0073. Recuperado de http://cgrfiles.cgr.go.cr/publico/ docs_cgr/2020/SIGYD_D_2020002020.pdf

Corrales, G. (2020, Abril 4). Un llamado a la cordura en los «Tiempos del COVID-19». La Revista.cr. Recuperado de https://www.larevista.cr/ gerardo-corrales-un-llamado-a-la-cordura-en-los-tiempos-del-covid-19/ 
Oviedo, E. (2020, Marzo 19). Gobierno propone destinar C1 billón a atender efectos de emergencia por covid-19. La Nación. Recuperado de https://www.nacion.com/el-pais/politica/ gobierno-pretende-destinar-1-billon-a-atender/XSBLAPE46VBQJAFOK5TRIVUOZM/story/

Leitón, P. (2020, Abril 30). Gobierno proyecta bajar histórico déficit a 8.6\% del PIB, pero requiere aprobación de nuevas leyes. La Nación. Recuperado de https://www.nacion. com/economia/politica-economica/gobierno-proyecta-bajar-historico-deficit-a-8/ TKGXDL6NMVHQHJHU7SONATEAYA/story/

Ministerio de Hacienda. (2019). Marco fiscal presupuestario de mediano plazo 2019-2023. Recuperado de https://www.hacienda.go.cr/docs/5d6713c34036d_Marco\%20Fiscal\%20Mediano\%20Plazo\%202019-2023.pdf

Casa Presidencial de Costa Rica. (2020). Decreto fija parámetros para la aplicación de la Regla Fiscal. En Comunicados. Recuperado de https://www.presidencia.go.cr/comunicados/2020/02/ decreto-fija-parametros-para-la-aplicacion-de-la-regla-fiscal/

Estrada P., M. F. (2020, Abril 24). Economía de Costa Rica decrecerá un 3,6\% en el 2020 debido al COVID-19, según Banco Central. Semanario Universidad. Recuperado de https://semanariouniversidad.com/ultima-hora/economia-de-costa-rica-decrecera-un-36-en-el-2020-debido-al-covid-19-segun-banco-central/

Thomsen, P. (2020, Marzo 30). Europe's COVID-19 Crisis and the Fund's Response. En INFBlog. Recuperado de https://blogs.imf.org/2020/03/30/europes-covid-19-crisis-and-the-fundsresponse/?utm_medium=email\&utm_source=govdelivery 